\title{
Species distribution and antifungal susceptibility patterns of Candida isolates from a public tertiary teaching hospital in the Eastern Cape Province, South Africa
}

\author{
P. Mnge ${ }^{1}$, B.I. Okeleye ${ }^{1,3}$, S.D. Vasaikar ${ }^{1,2}$ and T. Apalata ${ }^{1,2}$ \\ ${ }^{1}$ Division of Medical Microbiology, Department of Pathology and Laboratory Medicine, Faculty of Health Sciences, \\ Walter Sisulu University, Mthatha, South Africa \\ ${ }^{2}$ National Health Laboratory Services, Mthatha, South Africa \\ ${ }^{3}$ Phytomedicine and Phytopharmacology Research Group, Department of Plant Science, University of the Free State, \\ Phuthaditjhaba, South Africa
}

\begin{abstract}
Candida species are the leading cause of invasive fungal infections, and over the past decade there has been an increased isolation of drug resistant Candida species. This study aimed to identify the species distribution of Candida isolates and to determine their unique antifungal susceptibility and resistance patterns. During a cross-sectional study, 209 Candida isolates (recovered from 206 clinical samples) were collected and their species distribution was determined using ChromAgar Candida. The Vitek-2 system (Biomerieux, South Africa) was used to determine minimum inhibitory concentrations (MICs) to azoles (fluconazole, voriconazole), echinocandins (caspofungin, micafungin), polyenes (amphotericin B) and flucytosine. Four species of Candida were isolated, of which $C$. albicans was the most frequent, isolated in $45.4 \%(95 / 209)$ of the isolates, followed by C. glabrata: $31.1 \%(65 / 209)$. The MICs of the different antifungal drugs varied amongst the species of Candida. From the 130 isolates tested for MICs, $90.77 \%(112 / 130)$ were susceptible to all antifungal drugs and $6.9 \%(9 / 130)$ of the isolates were multi-drug resistant. $C$. dubliniensis $(n=2)$ isolates were susceptible to all the above mentioned antifungal drugs. There was no significant difference in species distribution amongst clinical specimens and between patients' genders $(P>0.05)$. An increase in MIC values for fluconazole and flucytosine towards the resistance range was observed. To our knowledge, this is the first report on surveillance of Candida species distribution and antifungal susceptibility at a public tertiary teaching hospital in Eastern Cape, South Africa.
\end{abstract}

Key words: Candida species; Distribution; Antifungal susceptibility; Identification; South Africa

\section{Introduction}

Candida species are commensal fungi of the human gastrointestinal tract, lower genital tract and mouth cavity. Among immunocompetent individuals, Candida species have an inherently low virulence. The incidence of candidiasis is more frequent in immunocompromised patients with impaired physiological and cellular barriers. Candida species colonize and invade host tissues wherein they cause localized to invasive systemic infections, which disseminate hematogenously to various organs of the body (1). Candida species are the leading cause of mycoses worldwide and the fourth leading cause of invasive nosocomial bloodstream infections with significant crude mortality and morbidity rates (2-5). In South Africa, the incidence and prevalence of Candida species is not well documented, however $C$. albicans remains the leading cause of candidiasis worldwide (6-9). Recent epidemiological reports indicate a change in species distribution patterns of Candida infections, with an increasing frequency of non-Albicans Candida species such as C. glabrata and C. parapsilosis being isolated from clinical samples $(10,11)$.

In African health care settings, amphotericin B and fluconazole are routinely used to treat Candida infections $(9,12)$. The changes in the epidemiology of Candida species is in parallel with the emergence of antifungal drug resistant species. Antifungal drug resistance is associated with an uncontrolled distribution and prolonged use of antifungals to treat recurrent infections in immunocompromised patients $(13,14)$. Furthermore, as it is imperative for

Correspondence: B.I. Okeleye: <ben_okeleye2005@yahoo.com> 
laboratories to provide identification to the species level, there is continued isolation of new species which are resistant to currently available antifungals.

The growing trend in antifungal drug resistance and emergence of new species of Candida, poses a need for regional surveillance of antifungal drug susceptibility profiles, since in vitro drug susceptibility patterns are associated with therapeutic outcome. This study sought to determine the distribution and in vitro susceptibility profiles of Candida species isolated from patients attending Nelson Mandela Academic Complex, a public tertiary teaching hospital in Mthatha, Eastern Cape.

\section{Material and Methods}

\section{Study population and sampling strategy}

A total of 209 Candida isolates (from 206 clinical samples) were collected during a cross-sectional study among patients at Nelson Mandela Academic Complex in Mthatha. A standardized data collection form was used to collect information on patients' demographics (age and gender) and clinical history from medical records. Permission to collect patient's clinical data, including laboratory information, was obtained from the hospital and laboratory managers. Ethical clearance was obtained from the Research Ethics Committee of Walter Sisulu University (Ethics Ref. No. 038/13).

\section{Isolation and identification of Candida species}

Yeast cells isolated from clinical samples were stored in $10 \%$ glycerol (Sigma-Aldrich, South Africa) at $-20^{\circ} \mathrm{C}$ until further use. Isolates were sub-cultured onto freshly prepared Sabaroud dextrose (SAB) agar and incubated overnight at $37^{\circ} \mathrm{C}$. The germ tube test was used for the presumptive identification of Candida species. Briefly, 24-h fresh cultures were inoculated on 3 drops of human serum and incubated at $37^{\circ} \mathrm{C}$. After $2.5 \mathrm{~h}$, the formation of germ tubes was observed under microscopy $(40 \times$ objective).

The ability of Candida species to form differentially colored colonies on chromogenic assay (ChromAgar Candida, Media-mage, South Africa) was used to identify Candida isolates to the species level. Inocula from 24-48 h SAB-agar cultures were re-cultured onto commercially prepared ChromAgar Candida plates and incubated for 48-72 h at $37^{\circ} \mathrm{C}$. Intense colony coloration was observed after incubation and species differentiation was done according to the manufacturer's instructions $(14,15)$.

\section{Antifungal susceptibility assay}

The antifungal susceptibility profile of Candida species was determined using the Vitek 2 Systems Version 07.01 (Biomeriex, South Africa) following the CLSI document M27-A3 (2015) (16). The antifungal agents tested were amphotericin B, fluconazole, voriconazole, caspofungin, micafungin and flucytosine. The test was carried out according to the manufacturer's instructions. About 2-3 colonies of 24-h Candida cultures were inoculated into 5 - $\mathrm{mL}$ glass tubes containing $3 \mathrm{~mL}$ of $10 \%$ saline, adjusted to 2 McFarland standards. Vitek 2 cards with 12 -fold serial dilutions of antifungals were placed onto the test tube and loaded onto the Vitek cassette. Loaded cassettes were then placed onto the Vitek instrument and incubated for 9 to $33 \mathrm{~h}$ depending on the sample. Standard strains C. albicans ATCC 90028 and C. parapsilosis ATCC 22019 were used for quality control. The antifungal susceptibility of the isolates was interpreted as sensitive (S), intermediate (I), and resistant (R) according to the CLSI interpretative breakpoints criteria $(16,17)$. The chi-square test was used to test for significant associations between the defined variables, while ANOVA was used to test the difference between groups. SPSS (IMB, USA) version 20.0 for Windows was used for all statistical analyses.

\section{Results}

\section{Species distribution}

A total of 209 isolates of Candida were obtained from 206 clinical specimens; the highest number of isolates were from urine specimens $(46.5 \%, n=97)$, followed by vaginal swabs $(30.6 \%, n=64)$. The mean age of the patients was $29.7 \pm 1.97$ years, ranging from 1 month to 87 years. The gender distribution of patients, based on clinical records was $148(71.9 \%)$ females and $46(22.3 \%)$ males. For $5.8 \%(12 / 206)$ isolates, the gender was not stated in clinical records. C. albicans accounted for 45.5\% (95/209) of the species isolated while $31.1 \%(65 / 209)$ of the species were C. glabrata, $12.4 \%(26 / 209)$ C. tropicalis, and C. dubliniensis accounted for $11.0 \%$ (23/209) of the total isolates (Table 1). There was no significant difference in species distribution amongst clinical specimens $\left(X^{2}=36\right.$; $\mathrm{DF}=66$, and $\mathrm{P}=0.99$ ) and between patients' genders $\left(X^{2}=11.964 ; D F=22\right.$, and $\left.P=0.958\right)$.

\section{Antifungal susceptibility testing}

The minimum inhibitory concentrations (MICs) and antifungal susceptibility of Candida species to the various antifungal drugs are summarized in Tables 2 and 3 . The results are presented by species as cumulative counts of susceptible organisms at each concentration throughout the full dilution series.

For C. albicans, resistance to fluconazole was $4.6 \%$ $(5 / 109$ at $\mathrm{MIC} \geqslant 64 \mu \mathrm{g} / \mathrm{mL})$, voriconazole resistance was $2.8 \%(3 / 109$, MIC $\geqslant 8 \mu \mathrm{g} / \mathrm{mL})$, caspofungin and micafungin resistance were $3.7 \%$ (4/109 at MIC $\geqslant 4 \mu \mathrm{g} / \mathrm{mL})$. In addition, voriconazole resistance was $2.8 \%$ (3/109, MIC $\geqslant 8 \mu \mathrm{g} / \mathrm{mL}$ ) and flucytosine resistance was $3.7 \%$ (4/109, MIC $\geqslant 32 \mu \mathrm{g} / \mathrm{mL}$ ). Intermediate resistance to flucytosine (MIC=8 $\mu \mathrm{g} / \mathrm{mL}$ ) and fluconazole $(\mathrm{MIC}=16 \mu \mathrm{g} / \mathrm{mL})$ was observed in 2 isolates of $C$. albicans and 1 other isolate exhibited intermediate resistance to voriconazole at MIC of $2 \mu \mathrm{g} / \mathrm{mL}$. 
Table 1. Distribution of Candida species among clinical specimens $(n=209)$.

\begin{tabular}{|c|c|c|c|c|c|c|}
\hline Specimen & C. albicans & C. glabrata & C. tropicalis & C. dubliniensis & $\mathrm{n}$ & Percentage (\%) \\
\hline Urine & 44 & 32 & 12 & 9 & 97 & $46.5 \%$ \\
\hline Vaginal swab & 24 & 19 & 11 & 10 & 64 & $30.6 \%$ \\
\hline Sputum & 14 & 6 & 1 & 2 & 23 & $11.0 \%$ \\
\hline Blood culture & 10 & 5 & 1 & 0 & 16 & $7.7 \%$ \\
\hline Pus & 2 & 0 & 0 & 1 & 3 & $1.4 \%$ \\
\hline Unknown & 1 & 1 & 1 & 0 & 3 & $1.4 \%$ \\
\hline Ascetic fluid & 0 & 2 & 0 & 1 & 3 & $1.4 \%$ \\
\hline
\end{tabular}

Table 2. Minimum inhibitory concentrations (MIC) of clinical isolates of Candida $(n=130)$ to various antifungal drugs.

\begin{tabular}{|c|c|c|c|c|c|c|c|c|c|c|c|}
\hline \multirow[t]{2}{*}{ Antifungal } & \multicolumn{11}{|c|}{ MIC range $(\mu \mathrm{g} / \mathrm{mL})^{*}$} \\
\hline & 0.065 & 0.125 & 0.25 & 0.5 & 1 & 2 & 4 & 8 & 16 & 32 & 64 \\
\hline \multicolumn{12}{|l|}{ Fluconazole } \\
\hline C. albicans & & & & & 84 & 4 & 4 & 10 & 1 & & 6 \\
\hline C. glabrata & & & & & 5 & & 3 & 1 & & & 2 \\
\hline C. tropicalis & & & & & 5 & & & 2 & & & 2 \\
\hline C. dubliniensis & & & & & 2 & & & & & & \\
\hline \multicolumn{12}{|l|}{ Voriconazole } \\
\hline C. albicans & & 96 & 4 & 1 & 4 & 1 & 1 & 2 & & & \\
\hline C. glabrata & & 9 & & & & & 2 & & & & \\
\hline C. tropicalis & & 7 & & & & & & 1 & 1 & & \\
\hline C. dubliniensis & & 2 & & & & & & & & & \\
\hline \multicolumn{12}{|l|}{ Caspofungin } \\
\hline C. albicans & & & 102 & & 3 & 4 & 4 & & & & \\
\hline C. glabrata & & & 9 & & & & 2 & & & & \\
\hline C. tropicalis & & & 6 & & & & 2 & 1 & & & \\
\hline C. dubliniensis & & & 2 & & & & & & & & \\
\hline \multicolumn{12}{|l|}{ Micafungin } \\
\hline C. albicans & 101 & 2 & & 2 & & & 4 & & & & \\
\hline C. glabrata & 6 & 3 & & & & & 2 & & & & \\
\hline C. tropicalis & 6 & 1 & & & & & 2 & & & & \\
\hline C. dubliniensis & 2 & & & & & & & & & & \\
\hline \multicolumn{12}{|l|}{ Amphotericin B } \\
\hline C. albicans & & & 72 & 31 & 2 & & 4 & & & & \\
\hline C. glabrata & & & 3 & 6 & & & & & 2 & & \\
\hline C. tropicalis & & & 6 & 1 & & & & & 2 & & \\
\hline C. dubliniensis & & & 2 & & & & & & & & \\
\hline \multicolumn{12}{|l|}{ Flucytosine } \\
\hline C. albicans & & & & & 103 & & & 2 & 2 & & 2 \\
\hline C. glabrata & & & & & 7 & 3 & & & & 1 & \\
\hline C. tropicalis & & & & & 6 & 1 & 1 & & & & 1 \\
\hline C. dubliniensis & & & & & 2 & & & & & & \\
\hline
\end{tabular}

* MIC breakpoints' interpretation: fluconazole susceptible: MIC $\leqslant 8 \mu \mathrm{g} / \mathrm{mL}$; fluconazole intermediate: MIC 16-32 $\mu \mathrm{g} / \mathrm{mL}$; fluconazole resistant: MIC $\geqslant 64 \mu \mathrm{g} / \mathrm{mL}$; micafungin and caspofungin susceptible: MIC $\leqslant 0.25$; micafungin and caspofungin intermediate: no clinical breakpoint; micafungin and caspofungin resistant: MIC $\geqslant 1 \mu \mathrm{g} / \mathrm{mL}$; flucytosine susceptible: MIC $\leqslant 4 \mu \mathrm{g} / \mathrm{mL}$; flucytosine intermediate: MIC 8-16 $\mu \mathrm{g} / \mathrm{mL}$; flucytosine resistant: MIC $\geqslant 32 \mu \mathrm{g} / \mathrm{mL}$; voriconazole susceptible: MIC $\leqslant 1 \mu \mathrm{g} / \mathrm{mL}$; voriconazole intermediate: MIC $2 \mu \mathrm{g} /$ $\mathrm{mL}$; voriconazole resistant: MIC $\geqslant 4 \mu \mathrm{g} / \mathrm{mL}$; amphotericin B susceptible: MIC $\leqslant 1 \mu \mathrm{g} / \mathrm{mL}$; amphotericin B Intermediate: no clinical breakpoint; amphotericin B resistant: MIC $>1 \mu \mathrm{g} / \mathrm{mL}$. 
Table 3. Antifungal susceptibility patterns of Candida species to different antifungal drugs.

\begin{tabular}{|c|c|c|c|c|c|}
\hline \multirow[t]{2}{*}{ Antifungal } & \multicolumn{5}{|c|}{ Species $(n=130)$} \\
\hline & $\begin{array}{c}\text { C. albicans } \\
(n=109)\end{array}$ & $\begin{array}{l}\text { C. glabrata } \\
(n=11)\end{array}$ & $\begin{array}{l}\text { C. tropicalis } \\
(\mathrm{n}=8)\end{array}$ & $\begin{array}{l}\text { C. dubliniensis } \\
(\mathrm{n}=2)\end{array}$ & $\mathrm{P}$ \\
\hline Fluconazole & & & & & 0.277 \\
\hline$S$ & $102(93.6 \%)$ & $9(81.8 \%)$ & $6(75 \%)$ & $2(100 \%)$ & \\
\hline 1 & $2(1.8 \%)$ & & & & \\
\hline $\mathrm{R}$ & $5(4.6 \%)$ & $2(18.2 \%)$ & $2(25 \%)$ & & \\
\hline Voriconazole & & & & & 0.061 \\
\hline$S$ & $105(96.3 \%)$ & $9(81.8 \%)$ & $6(75 \%)$ & $2(100 \%)$ & \\
\hline I & $1(0.9 \%)$ & & & & \\
\hline $\mathrm{R}$ & $3(2.8 \%)$ & $2(18.2 \%)$ & $2(25 \%)$ & & \\
\hline Caspofungin & & & & & 0.065 \\
\hline $\mathrm{s}$ & $105(96.3 \%)$ & $9(81.8 \%)$ & $6(75 \%)$ & $2(100 \%)$ & \\
\hline $\mathrm{R}$ & $4(3.7 \%)$ & $2(18.2 \%)$ & $2(25 \%)$ & & \\
\hline Micafungin & & & & & 0.068 \\
\hline$S$ & $105(96.3 \%)$ & $9(81.8 \%)$ & $6(75 \%)$ & $2(100 \%)$ & \\
\hline $\mathrm{R}$ & $4(3.7 \%)$ & $2(18.2 \%)$ & $2(25 \%)$ & & \\
\hline Amphotericin B & & & & & 0.068 \\
\hline S & $105(96.3 \%)$ & $9(81.8 \%)$ & $6(75 \%)$ & $2(100 \%)$ & \\
\hline $\mathrm{R}$ & $4(3.7 \%)$ & $2(18.2 \%)$ & $2(25 \%)$ & & \\
\hline Flucytosine & & & & & 0.0934 \\
\hline S & $102(93.6 \%)$ & $10(90.9 \%)$ & $7(87.5 \%)$ & $2(100 \%)$ & \\
\hline I & $2(1.8 \%)$ & & & & \\
\hline $\mathrm{R}$ & $5(4.6 \%)$ & $1(9.1 \%)$ & $1(12.5 \%)$ & & \\
\hline
\end{tabular}

S: susceptible; I: intermediate; R: resistant. $\mathrm{P}<0.05$ (chi-square test).

A similar resistance pattern was observed in 2 isolates of both C. glabrata and C. tropicalis. One isolate from each species was fully resistant to all antifungal drugs, while the other isolate was resistant to all antifungal drugs except for flucytosine at MIC $\geqslant 64 \mu \mathrm{g} / \mathrm{mL}$ in C. tropicalis and MIC of $32 \mu \mathrm{g} / \mathrm{mL}$ in C. glabrata. Notably, at flucytosine MIC of $\leqslant 1 \mu \mathrm{g} / \mathrm{mL}$ (susceptible), $3(2.6 \%)$ C. albicans and $2(18.2 \%)$ C. glabrata isolates were resistant to all other classes of antifungals. Two isolates of $C$. albicans were resistant to all antifungals, except flucytosine (MIC $\leqslant 16 \mu \mathrm{g} / \mathrm{mL}$ ) and intermediate to voriconazole at MIC of $2 \mu \mathrm{g} / \mathrm{mL}$.

\section{Discussion}

The study sought to compare the species distribution pattern of Candida and to compare the antifungal susceptibility patterns of 6 antifungal drugs against the isolated species of Candida. The most common species isolated was $C$. albicans, and this finding is similar to previously published reports in South Africa, where C. albicans was the most commonly isolated species from clinical samples $(9,12)$. In a study that determined the microbial carriage in bloodstream infections, $C$. albicans was responsible for $31 \%(21 / 68)$ of all fungal infections (18). The findings of this study also correlate with reports from the Neonatal Intensive Care Unit in Dr. George Mukhari Hospital, South
Africa, where C. albicans was the most common species isolated at the unit in 2002 (9). A recent report by Makhado et al. (9) has, however, indicated a shift in the epidemiology of candidiasis with C. krusei replacing C. albicans as the most common species isolated. Although $C$. albicans was the most common species isolated in this present study, the rate of non-albicans Candida species varied amongst the clinical specimens and these species were isolated in more than $50 \%$ of the clinical samples.

In contrast to $C$. albicans being the most commonly isolated species, C. glabrata, C. krusei and C. tropicalis are also reported to be leading causes of candidiasis. The frequency of these non-albicans Candida species varies throughout the world. This variation may be a result of the underlying medical condition, geographic distribution and patients' age and gender (1,19-21). C. glabrata and C. tropicalis are the most common species isolated in neutropenic patients, in catheter related infections, and in adults, while $C$. krusei has been reported as the leading cause of candidemia. The increase in isolation of nonalbicans Candida species is in parallel with a decrease in the isolation of $C$. albicans. $(1,14,18,20)$

The high frequency of Candida species isolated from vaginal and urine samples from female patients can be explained by the imbalance in vaginal microflora as a result of diabetes and vaginal estrogenization, which in turns 
gives rise to vulvovaginal candidiasis $(21,22)$. Symptomatic vulvovaginal candidiasis (VVC) occurs in women aged 18-84 years and is associated with a significant morbidity rate. Approximately $75 \%$ of women experience one episode of VVC in their lifetime (23-25). The underlying medical conditions for the isolates were vaginal discharge, retroviral disease and pelvic inflammatory discharge. On most urine samples, the clinical diagnosis was stated as illegible thus making it difficult to ascertain if the presence of yeast in urine represents a true infection or merely colonization and contamination of the bladder (26).

The ongoing change in the epidemiology of Candida species is in parallel with the emergence of antifungal drug resistant Candida species (14). This is especially true for fluconazole, which is used as the first line drug treatment for hematological malignancy, HIVIAIDS and oropharyngeal candidiasis in South Africa and Africa as a whole. This, in turn, leads to the selection of less susceptible isolates, with an inherent or acquired resistance to fluconazole, especially C. albicans and C. tropicalis isolates $(8,9,12,13,27)$. The widespread use of fluconazole does not only lead to selection of antifungal drug resistant species, but also to a shift from C. albicans as the leading cause of candidiasis to nonalbicans species such as $C$. glabrata and $C$. tropicalis as the causative agents of Candida infections $(14,28)$.

Relatively low levels of fluconazole resistance were observed in all species of Candida isolated; it is worth mentioning that the non-albicans species had the highest levels of resistance compared to $C$. albicans. Primary resistance to fluconazole is reported in C. albicans, C. tropicalis and C. krusei isolates. Multi-drug resistance was observed in C. albicans, C. glabrata and C. tropicalis isolates, indicating a major public health concern and reflecting the inappropriate use of antifungals drugs. This also supports the importance of ongoing in vitro surveillance and careful monitoring of antifungal treatment regimens since in vitro resistance is associated with therapeutic failure $(12,14,29)$.

The lowest MIC $(0.065 \mu \mathrm{g} / \mathrm{mL})$ in all species of Candida was observed in micafungin (Table 2); this echinocandin class of antifungals is said to confer excellent fungicidal effects against Candida species. Echinocandins are not routinely used in antifungal therapy due to their hepatic effect. Flucytosine resistance, more especially in C. glabrata isolates, is reported when the drug is administered as monotherapy. It is, therefore, suggested that in order to achieve optimal flucytosine activity, the drug should be administered in combination with amphotericin $\mathrm{B}$, and/or any azole and echinocandins (30-32).

\section{References}

1. Quindos G. Epidemiology of candidemia and Invasive candidiasis changing face. Rev Iberoamer Micol 2013; 271: 1-7.
The antifungal susceptibility of echinocandins and liposomal amphotericin B against Candida biofilms was investigated by Marcos-Zambrano (33). It was found that C. tropicalis biofilms had the highest level of resistance and amphotericin B was unable to reduce the metabolic activity of the biofilms. In a study conducted by Blignaut et al. (34), the South African clade of $C$. albicans had an $8.4 \%$ level of resistance to amphotericin B and the clade was found to be naturally resistant to that drug. That finding is supported in this study, in which C. tropicalis isolates had a $25 \%$ level of resistance compared to $C$. albicans and C. glabrata. Although the results are statistically not significant, this finding has to be considered when amphotericin B is used to treat $C$. tropicalis infections. This is equally important, since amphotericin $B$ is routinely used to treat fluconazole-resistant infections (33).

There was a degree of variability in the MIC values within the same class of antifungals, for example the MIC range for micafungin was between 0.025 to $4 \mu \mathrm{g} / \mathrm{mL}$ and caspofungin MIC ranged from 0.065 to $8 \mu \mathrm{g} / \mathrm{mL}$. On the other hand, MIC values for fluconazole ranged from 1 to $64 \mu \mathrm{g} / \mathrm{mL}$ and for voriconazole from 0.125 to $16 \mu \mathrm{g} / \mathrm{mL}$. A similar pattern was also observed by Villareal et al. (35) in C. glabrata isolates. The differences in MIC values within species and classes are indicative of species-specific resistance patterns. Hence, there is a need for regional surveillance of fungal species distribution, and antifungal therapeutic regimes should be implemented based on epidemiological data and antifungal sensitivity. The low MICs and increased spectrum of activity for voriconazole and micafungin in C. albicans and non-albicans species suggest good clinical activity for these drugs.

The epidemiology of Candida infections in Africa, a home for new and emerging drug-resistant Candida species, is not well documented $(9,14)$, and this study presented the first regional surveillance in the Eastern Cape province to investigate the prevalence and antifungal susceptibility patterns of Candida isolates.

\section{Acknowledgments}

We would like to thank the staff of National Health Laboratory Services (Mthatha) for their support in sample collection, Mrs. N. Sibathathu for her technical assistance in conducting laboratory experiments, the National Research Foundation (South Africa) for financial support, and lastly the staff of Division of Medical Microbiology, Department of Pathology and Laboratory Medicine (Walter Sisulu University) for assisting the successful completion of the study.

2. Madhavan P, Jamal F, Chang PP. Laboratory isolation and identification of Candida species. J App/ Sci 2011; 11: 2870 2877, doi: 10.3923/jas.2011.2870.2877. 
3. Sanglard D, Kuchler K, Fischer F, Pagani JL, Monod M, Bille $\mathrm{J}$. Mechanisms of resistance to azole antifungal agents in Candida albicans isolates from AIDS patients involves specific multidrug transporters. Antimicrob Agents Chemother 1995; 39: 2378-2386, doi: 10.1128/AAC.39.11.2378.

4. Bruder-Nascimento $A$, Camargo $\mathrm{CH}$, Sugizak MF, Sadatsune $\mathrm{T}$, Montelli AC, Mondelli AL, et al. Species distribution and susceptibility profile of Candida species in a Brazilian public tertiary hospital. BMC Res Notes 2010; 3: 1-5, doi: 10.1186/ 1756-0500-3-1.

5. Bhooshon S, Gayal A, Agrawa IA, Verma V. Prevalence and drug resistant of Candida species in pediatrics patients in Tertiary Care Hospital, North India. J Microbiol Biomed Res 2015; 1: 1-6.

6. Okonko IO, Odu NN, Kolade A, Nwanze JC. Detection and prevalence of Candida isolates among patients in Ibadan, Southwestern Nigeria. J Microbiol Biotechnol Res 2011; 1: 176-184.

7. Pfaller MA, Diekama DJ. Epidemiology of invasive candidiasis: A persistent public health problem. Clin Microbiol Rev 2007; 20: 133-163, doi: 10.1128/CMR.00029-06.

8. Owatade FJ, Gulube Z, Ramla S, Patel M. Antifungal susceptibility of Candida albicans isolated from the oral cavities of patients with HIV infection and cancer. South African Dental J 2016; 71: 8-11.

9. Makhado NA, Ismal F, Dango Y, Chephe TJH, Hoosen AA, Chabeleng M. Antifungal susceptibility profile of yeast isolates from sterile sites at a public teaching hospital in South Africa. South African J Infect Dis 2014;29: 97-100.

10. Nnadi NE, Ayanbimpe GM, Scordino F, Okolo MO, Enwean $\mathrm{IB}$, Crisea $\mathrm{G}$, et al. Isolation and molecular characterization of C. Africana from Jos, Nigeria. Med Mycol 2012: 50: 765-767, doi: 10.3109/13693786.2012.662598.

11. Chow JK, Golan $Y$, Ruthezer R, Karchmer AW, Carmeli $Y$, Lichtenberg $D$, et al. Factors associated with candidemia caused by non-albicans species and Candida albicans in the intensive care unit. Clin Infect Dis 2008; 46:1206-1213, doi: $10.1086 / 529435$.

12. Abrantes PMDS, McArthur CP, African CWJ. Multi-drug resistance (MDR) oral Candida species isolated from HIVpositive patients in South Africa and Cameroon. Diag Microbiol Infect Dis 2014; 79: 222-227, doi: 10.1016/j.diagmicro bio.2013.09.016.

13. Hajjeh RA, Sofar AN, Harrison LH, Lyon GM, HartingtonSkaggs BA, Mirza SA, et al. Incidence of bloodstream infections due to Candida species and in vitro susceptibilities of isolates collected from 1998 to 2000 in a population-based active surveillance program. J Clin Microbiol 2004; 42: 1519-1527, doi: 10.1128/JCM.42.4.1519-1527.2004.

14. Efushnie AM, Oduyebo O, Osuagwu CS, Koenig B. Species distribution and antifungal susceptibility of Candida Isolates from pregnant women in a tertiary hospital in nigeria. African J Clin Exper Microbiol 2016; 17: 183-189, doi: 10.4314/ ajcem.v17i3.5.

15. ChromAgar: The Chromogenic Media Pioneer. NT-EXT-001. Version 7.

16. Wayne PA. Reference method for broth dilution antifungal susceptibility testing of yeasts. Clin Lab Standards Inst 2008; 3: M27-A3.

17. Behzadi P, Behzadi E, Ranjbar R. Urinary tract infections and C. albicans. Centr Eur J Eurol 2015; 68: 96-101.
18. Dramowski A, Cotton MF, Rabie H, Whitelaw A. Trends in paediatric bloodstream infections at a South African referral hospital. BMC Paediatrics 2015; 15: 33-44, doi: 10.1186/ s12887-015-0354-3.

19. Olaniran O, Adefusi OF, Idowu OJ, Oladipo OA, Afolayan DO, Aderibigbe I, et al. Isolation and evaluation Candida species of among pregnant women in Obafemi Awolowo University teaching hospital, ile-ife. Nigeria. J. Clin Microbiol Case Reports 2015; 1: 1-6.

20. Fortún J, Martin-Dávila P, Pedrosa EG, Pintado V, Cobo T, et al. Emerging trends in candidemia: A higher incidence but a similar outcome. J. Infect 2012, 65: 64-70, doi: 10.1016/j. jinf.2012.02.011.

21. Yapur N. Epidemiology and risk factors for invasive candidiasis. Ther Clin Risk Manag 2014; 10: 95-105, doi: 10.2147/TCRM.S40160.

22. Mendling W, Brasch J. Guideline vulvovaginal candidosis (2010) of the German society for gynecology and obstetrics, the working group for infections and infectimmunology in gynecology and obstetrics, the germansociety of dermatology, the board of german dermatologists and the german speaking mycological society. Mycoses 2012; 55: 1-13, doi: 10.1111/j.1439-0507.2012.02185.x.

23. Shrivastav VK, Shukla D, Shrivastav A, Jana AM. Prevalence of vaginal candidiasis in diabetic women of Madhya Pradesh, India. Int J Current Microbiol Appl Sci 2015; 4: 834-846

24. Apalata T, Carr WH, Sturm WA, Longo-Mbenza B, Moodley $P$. Factors associated with symptomatic vulvovaginal candidiasis: A study among women attending a primary healthcare clinic in Kwazulu-Natal, South Africa. Ann Med Health Sci Res 2014; 3: 410-416, doi: 10.4103/2141-9248. 133470.

25. Oyewole OA, Okoliegbe IN, Alkhalil S, Isah P. Prevalence of vaginal candidiasis among pregnant women attending Federal University of Technology, Minna, Nigeria, Bosso Clinic. Res J Pharm Biol Chel Sci 2013; 4: 113-120.

26. Nucci M, Colombo AL. Emergence of resistant Candida in neutropenic patients. Braz J Infect Dis 2002; 6: 124-128, doi: 10.1590/S1413-86702002000300004.

27. Sebti A, Kiem TE, Peili D, Cathurvedi V, Wong M, Doney A, et al. Candida dubliniensis at a cancer. Clin Infect Dis 2001; 32: 1034-1038, doi: 10.1086/319599.

28. Perlin DS, Shor E, Zhao Y. Update on antifungal drug resistance. Curr Clin Micro Rpt 2015; 2: 84-95, doi: 10.1007/ s40588-015-0015-1.

29. Mulu A, Kassu A, Anagaw B, Moges B, Gelaw A et al. Frequent detection of 'azole' resistant Candida species among late presenting AIDS patients in northwest Ethiopia. BMC Infect Dis 2013; 13: 1-10, doi: 10.1186/1471-2334-13-82.

30. Mukherjez P, Wang M. Antifungal drug resistance: significance and mechanisms. Informa Healthcare 2010: 63-86.

31. Charlier C, Sissy CEL, Bachelier-Bassi S, Scemla A, Quesne G, Sitterlé E, et al. Acquired flucytosine resistance during combination therapy with caspofungin and flucytosine for Candida glabrata cystitis. Antimicrob Agents Chemother 2016; 60: 662-665, doi: 10.1128/AAC.02265-15.

32. Locke JB, AlmaguerAL, Zuill DE, Bartizal K. Characterization of in vitro resistance development to the novel echinocandin CD101 in Candida species. Antimicrob Agents Chemother 2016; 60: 6100-6107, doi: 10.1128/AAC.00620-16. 
33. Marcos-Zambrano LJ, Gómez-Perosanz M, Escribano P, Zaragoza O, Bouza E, Guinea J. Echinocandin-resistant yeast species: biofilm production and anti-biofilm activity of echinocandins and liposomal amphotericin B. Antimicrob Agents Chemother 2015:1-28.

34. Blignaut E, Molepo J, Pujol C, Soll DR, Pfaller MA. Claderelated amphotericin $B$ resistance among South African
Candida albicans isolates. Diagn Microbiol Infect Dis 2005; 53: 29-31, doi: 10.1016/j.diagmicrobio.2005.03.013.

35. Villareal NC, Fothergill AW, Kelly C, et al. Candida glabrata resistance to caspofungin during therapy. 44th Interscience Conference on Antimicrobial Agents and Chemotherapy. Washington: American Society for Microbiology; 2004. 